\title{
Height and Risk of Heart Failure in the Physicians' Health Study I
}

\author{
Akintunde $O$ Akinkuolie, MD, MPH ${ }^{\mathrm{a}}$, Megan Aleardi, $\mathrm{MPH}^{\mathrm{b}}$, Ajibade $\mathrm{O}$ Ashaye, MD, MPH${ }^{\mathrm{c}}, \mathrm{J}$. \\ Michael Gaziano, Md ${ }^{a, d}$, and Luc Djousse, MD, ScD ${ }^{a, d}$ \\ aBrigham and Women's Hospital and Harvard Medical School \\ bMassachusetts General Hospital \\ 'Evidence-Based Practice Center, UCONN/Hartford Hospital, Hartford, CT \\ ${ }^{\mathrm{d} B o s t o n}$ VA Healthcare Systems, Boston, MA
}

\begin{abstract}
While previous studies have reported an association between height and cardiovascular disease, it is unclear whether height is associated with the risk of heart failure (HF). We hypothesized that height will be inversely associated with HF risk. We used prospective data from 22042 male physicians (mean age 53.8 years) from the Physicians' Health Study I. Height was self-reported at baseline. Incident HF was ascertained via follow-up questionnaires and validated through review of medical records in a subsample. Cox proportional hazard model was used to compute hazard ratios (HR) with corresponding 95\% confidence intervals (CI). Mean height ( \pm standard deviation) was $1.78(0.07) \mathrm{m}$. A total of $1444 \mathrm{HF}$ cases occurred during a mean follow-up of 22.3 years. Compared to subjects in the lowest height category $(1.40-1.73 \mathrm{~m}), \mathrm{HR}(95 \% \mathrm{CI})$ for HF were $0.86(0.74-0.99), 0.82(0.70-0.95)$ and $0.76(0.63-0.91)$ for height categories of 1.74-1.78 m, 1.79-1.83 m, and 1.84-2.08 m, respectively, after adjustment for age, weight, hypertension, and diabetes mellitus ( $p$ trend $=0.0023)$. HR $(95 \%$ CI) per SD increment in height was 0.92(0.86-0.98) in a fully adjusted model. Exclusion of individuals with prevalent atrial fibrillation, left ventricular hypertrophy, valvular heart disease, and a history of coronary artery bypass graft, yielded similar results [HR per SD: $0.88(0.83-0.94)]$. In conclusion, our data demonstrated an inverse association between height and incident HF in US male physicians. Additional studies to elucidate underlying biologic mechanisms are warranted.
\end{abstract}

\section{Keywords}

Heart Failure; Stature; Height; Epidemiology

The effects of gravity on the cardiovascular system of an upright individual are predominantly due to a reduction in vascular pressure as well as an increased compliance within the venous system, resulting in the translocation of vascular volume away from the

(C) 2011 Excerpta Medica, Inc. All rights reserved.

Address correspondence to: Akintunde O Akinkuolie, Center for cardiovascular disease prevention, Division of Preventive Medicine, Brigham and Women's Hospital, 900 Commonwealth Avenue East, 3rd Floor, Boston, Massachusetts 02215. Tel: (857)-719-2351 Fax: (617) 525-7739 AOAKINKUOLIE@PARTNERS.ORG.

Publisher's Disclaimer: This is a PDF file of an unedited manuscript that has been accepted for publication. As a service to our customers we are providing this early version of the manuscript. The manuscript will undergo copyediting, typesetting, and review of the resulting proof before it is published in its final citable form. Please note that during the production process errors may be discovered which could affect the content, and all legal disclaimers that apply to the journal pertain.

Institute and location where work was performed: Brigham and Women's Hospital, Boston, MA

Disclosures None 
heart. Additionally, gravity also increases compliance of the arterial tree, giving rise to a reduced peripheral resistance and hence a reduction in afterload. Meanwhile the effect of gravity on the cardiovascular system increases as height increases ${ }^{1}$. Also, there are findings of an inverse association between height and pulse pressure (PP) ${ }^{2}$, which is a risk factor for HF. However, despite beneficial effects of height on HF risk factors such as coronary heart disease (CHD) and PP, no previous study has examined the relation between height and HF. Therefore, our objective was to determine whether adult height is inversely associated with the risk of HF in a prospective cohort of US male physicians.

\section{METHODS}

Data from the Physicians' Health Study (PHS) I was used in this analysis. A detailed description of the PHS I has been published previously ${ }^{3}$. In brief, this is a completed randomized controlled trial using a $2 \times 2$ factorial design of low dose aspiring and $\beta$ carotene in the primary prevention of CVD and cancer. Each participant gave a written informed consent, and the Institutional Review Board at the Brigham and Women's Hospital approved the study protocol. Of the 22071 participants recruited into the PHS I, 29 were excluded because of missing information on height $(n=6)$, baseline myocardial infarction $(n=1)$, and baseline HF $(n=22)$. Hence, a total sample of 22042 participants was used in present analyses.

At baseline, self-reported questionnaires were used to assess information on height. Height was reported in inches. We multiplied by a factor of 0.0254 to convert inches into meters (m).

Incident HF ascertainment in the PHS I has been achieved through the use of follow-up questionnaires. A questionnaire was mailed out every 6 months for the first year and yearly thereafter to obtain information on outcomes of interest, including heart failure. A detailed description of heart failure validation in the PHS has been published previously ${ }^{4}$. In the current analyses, heart failure and death ascertained through March 2010 was used.

Each participant provided information on weight, age, prevalent atrial fibrillation (yes or no), and hypertension based on reported blood pressure values or use of antihypertensive medications (yes or no), valvular heart disease (yes or no), history of coronary artery bypass graft (yes or no), diabetes mellitus (yes or no), regular vigorous exercise enough to break up sweat (yes or no), cigarette smoking (never, past or current) and alcohol consumption ( daily, weekly, monthly or rarely/never)

As we did not assume a linear association between height and HF, we initially created categories of height by using cut points that were close to the quartile distribution of height in this population $(<1.74,1.74-1.78,1.79-1.83$ and $>1.83 \mathrm{~m})$ for analysis. We presented baseline characteristics across categories of height. Person-time of follow up was computed from baseline until first occurrence of HF, death, or date of last known contact. Within each category of height, crude incidence rates were calculated as new HF cases divided by person-time follow up. We fitted Cox-proportional hazard models ${ }^{5}$ to estimate hazard ratios with corresponding $95 \% \mathrm{CI}$. Potential confounding was investigated by assessing whether the regression coefficient for height (continuous) changed by more than $10 \%$ when adding potential confounders to a model singly or jointly ${ }^{6}$. We considered factors that could influence height as well as influence the risk of HF in building our parsimonious model. We then tested the proportional hazard assumptions by including an interaction term between follow up time (log-transformed) and variables in our parsimonious model. When the proportional hazard assumption was violated $(\mathrm{P}<0.05)$ for a given variable, we thus accounted for the non proportionality of such variable by stratification ${ }^{5}$. 
The initial model (Model 1) adjusted for age (categorical) and weight (continuous) while the parsimonious model (Model 2) controlled for age (categorical), weight (continuous), hypertension (yes or no), and prevalent diabetes mellitus (yes or no). An additional model (Model 3) was then created to assess the impact of smoking, exercise and alcohol intake on the observed association between height and risk of HF.

In a sensitivity analysis, we repeated main analysis after exclusion of participants with conditions known to predispose to HF (prevalent atrial fibrillation, left ventricular hypertrophy, valvular heart disease and a history of coronary artery bypass graft). Probability value for linear trend was computed by fitting a continuous variable that assigned the median height in each height category in a Cox regression model. Linearity of the relationship between height and heart failure risk was satisfied upon visual inspection of a plot of martingale residuals. We then fitted height as a continuous variable and computed hazard ratios per each SD increment in height $(0.07 \mathrm{~m})$. All analysis were performed using SAS version 9.2 (SAS institute, Cary, NC). Significant level was set at 0.05 and all $\mathrm{P}$ values were 2 tailed $(\alpha=0.05)$.

\section{RESULTS}

Among the 22042 men in the PHS I with baseline information on height, the mean age at randomization was $53.8 \pm 9.5$ years (range 40 to 86 ) and mean height was $1.78 \mathrm{~m}$ (range 1.40 to 2.08). During a mean follow-up time of 22.26 years, a total of 1444 incident heart failure cases were reported in the study. Table 1 shows baseline characteristics of the participants according to categories of height. The incidence of HF ranged from 28.3/10,000person-years to $30.4 / 10,000$ person-years from the shortest to tallest height category. In multivariable Cox regression model, height was inversely associated with the risk of HF. We observed up to a $24 \%$ reduction in the risk of HF when the tallest category was compared to the shortest category of height; ( $\mathrm{P}$ for trend 0.0023) (Model 2, Table 2). For $1 \mathrm{SD}$ increment in height, the multivariable adjusted HR (95\% CI) for HF was 0.89(0.84-0.95). similar results were obtained after exclusion of prevalent atrial fibrillation, left ventricular hypertrophy, valvular heart disease and a history of coronary artery bypass graft : HR (95\% CI) for $1 \mathrm{SD}$ increment in height was $0.88(0.83-0.94)$. On evaluating the effect of modifiable behavioral risk factors (smoking, exercise and alcohol intake) on our observed result, a similar trend in the risk of HF across height categories was noted ( $\mathrm{p}$ trend $=0.038$ ).

\section{DISCUSSION}

In this study, we report for the first time an inverse relation between adult height and the incidence of $\mathrm{HF}$ in a large prospective cohort. Though no previous study has evaluated the association between height and $\mathrm{HF}$, several studies have reported an inverse relation between height and $\mathrm{CHD}^{7-14}$. The British Regional Heart study by walker $\mathrm{M}$ et al ${ }^{15}$ found that the risk of myocardial infarction was approximately two-fold higher in the shortest quintile when compared to the tallest quintile of height, even after controlling for age, socioeconomic status (SES), total cholesterol, HDL-cholesterol, systolic blood pressure, and cigarette smoking. Additionally, there is evidence of an inverse relation between height and traditional risk factors for CVD such as serum lipids and $\mathrm{PP}^{2,16,17}$. In one instance, Kouda $\mathrm{K}$. et al concluded that height velocity is inversely associated with dynamic changes in serum lipids ${ }^{17}$. Linked to this is evidence showing that the administration of growth hormone $(\mathrm{GH})$ decreases low density lipoprotein cholesterol (LDL-C) and increases high density lipoprotein cholesterol (HDL-C) ${ }^{18}$. These reports suggest that height attained at adulthood may be a marker for a cardioprotective lipid profile and a subsequent lower risk for HF. Also, PP, which is a predictor of congestive $\mathrm{HF}^{19}$, has been shown to have an inverse relation with height ${ }^{2}$. Other factors that may provide an indirect link to our results 
includes: lung function and SES. A direct relationship has previously been established between height and better lung function ${ }^{7}$. Meanwhile, data from the Framingham study showed that the risk of congestive HF increased progressively the lower the vital capacity ${ }^{20}$. Similarly, SES is known to be associated with the risk of congestive $\mathrm{HF}^{21}$, more so, since childhood SES is a major environmental influence of childhood growth, it has been implicated as an indicator of adult height ${ }^{10}$. However, The PHS I cohort is homogenous in terms of SES 22 thereby making it less likely for SES to serve as a potential source of residual confounding in the present study. Therefore, traditional CVD risk factors such as PP, lipids, and lung function may provide an explanation of our findings.

Based on the 2 major mechanisms underlying the development of HF - systolic and diastolic dysfunction ${ }^{23}$ - the effect of gravity on the cardiovascular system offers a biologically plausible mechanism to explain our observation of an inverse relationship between height and the incidence of $\mathrm{HF}^{1}$; gravity force increases the distensibility of the vascular tree ${ }^{24}$, leading to a reduced venous flow to the heart. Therefore, the effective preload may be reduced by gravity giving rise to a reduced systolic work on the heart. Also, the increased distensibility owing to gravity might reduce peripheral resistance and hence, enhance cardiac output. Thus, gravity might reduce systolic work of the heart and prevent the development of LVH. Langou et al reported that upright posture is associated with reduced ventricular chamber size, ventricular wall stress and myocardial oxygen demand ${ }^{25}$, underscoring the importance of gravity on the cardiovascular system. The hemodynamic relationship between PP and height also offers another plausible biological mechanism that may explain our results. Height determines the length of the arterial tree and a shorter arterial tree brings arterial pulse wave reflection sites closer to the heart. These reflected waves also return to the aorta earlier in systole giving rise to an augmentation of the primary waves which mimics the effect of aging on the arterial tree, thus, a shorter individual will require more pulsatile effort from the left ventricle; the net effect is a reduced compliance and an increased PP with shorter height ${ }^{26}$. As such, PP will reduce as height increases implying that arterial compliance increases with height. A reduced PP may translate into a lower risk of LVH and subsequently, a lower risk of HF.

Our study has limitations. First, generalizability of our data is limited by the fact that our participants were male physicians whose lifestyles may be different from men or the general population as a whole. Second, we did not account for possible changes in our exposure over time; however we believe that it is unlikely for a substantial change in height to have occurred in our cohort of adult males. Third, we did not have data on echocardiography measures of $\mathrm{LVH}$ to further explore the role of $\mathrm{LVH}$ as a potential mediator of height-HF relation. In addition, the potential role of PP as a mediating factor was not considered in the current analysis. Lastly, chance or residual confounding from unmeasured variables might explain our results. Nonetheless, our study benefitted from unique characteristic of the PHS I such as its large sample size, long follow-up, valid ascertainment of HF and ability to minimize the effect of SES.

\section{Acknowledgments}

We are indebted to the participants in the PHS for their outstanding commitment and cooperation and to the entire PHS staff for their expert and unfailing assistance.

Sources of Funding The PHS is supported by grants CA-34944 and CA-40360 from the National Cancer Institute and grants HL-26490 and HL-34595 from the National Heart, Lung, and Blood Institute, Bethesda, Md. Dr Djoussé is Principal Investigator on grant K01 HL-70444 from the National Heart, Lung, and Blood Institute, Bethesda, Md. 


\section{References}

1. Martin-Du Pan RCBR, Girardier L. The role of body position and gravity in the symptoms and treatment of various medical diseases. SWISS MED WKLY. 2004; 134:543-551. [PubMed: 15551157]

2. Langenberg C, Hardy R, Kuh D, Wadsworth ME. Influence of height, leg and trunk length on pulse pressure, systolic and diastolic blood pressure. J Hypertens. 2003; 21:537-543. [PubMed: 12640247]

3. Final report on the aspirin component of the ongoing Physicians' Health Study. Steering Committee of the Physicians' Health Study Research Group. N Engl J Med. 1989; 321:129-135. [PubMed: 2664509]

4. Djousse L, Driver JA, Gaziano JM. Relation between modifiable lifestyle factors and lifetime risk of heart failure. JAMA. 2009; 302:394-400. [PubMed: 19622818]

5. Cox D. Regression models and life tables. JR Stat Soc. 1972; 34:187-220.

6. Greenland, S.; Rothman, KJ. Introduction to stratified analysis Modern Epidemiology. Lippincott Williams \& Wilkins; Philadelphia: 1998. p. 225-255.

7. Cook NR, Hebert PR, Satterfield S, Taylor JO, Buring JE, Hennekens CH. Height, lung function, and mortality from cardiovascular disease among the elderly. Am J Epidemiol. 1994; 139:10661076. [PubMed: 8192139]

8. Hebert PR, Rich-Edwards JW, Manson JE, Ridker PM, Cook NR, O’Connor GT, Buring JE, Hennekens $\mathrm{CH}$. Height and incidence of cardiovascular disease in male physicians. Circulation. 1993; 88:1437-1443. [PubMed: 8403290]

9. Kannam JP, Levy D, Larson M, Wilson PW. Short stature and risk for mortality and cardiovascular disease events. The Framingham Heart Study. Circulation. 1994; 90:2241-2247. [PubMed: 7955180]

10. Langenberg C, Shipley MJ, Batty GD, Marmot MG. Adult socioeconomic position and the association between height and coronary heart disease mortality: findings from 33 years of followup in the Whitehall Study. Am J Public Health. 2005; 95:628-632. [PubMed: 15798120]

11. Marmot MG, Rose G, Shipley M, Hamilton PJ. Employment grade and coronary heart disease in British civil servants. J Epidemiol Community Health. 1978; 32:244-249. [PubMed: 744814]

12. Morris JN, Kagan A, Pattison DC, Gardner MJ. Incidence and prediction of ischaemic heartdisease in London busmen. Lancet. 1966; 2:553-559. [PubMed: 4161611]

13. Smith GD, Hart C, Blane D, Hole D. Adverse socioeconomic conditions in childhood and cause specific adult mortality: prospective observational study. BMJ. 1998; 316:1631-1635. [PubMed: 9603744]

14. Wannamethee SG, Shaper AG, Whincup PH, Walker M. Adult height, stroke, and coronary heart disease. Am J Epidemiol. 1998; 148:1069-1076. [PubMed: 9850129]

15. Walker M, Shaper AG, Phillips AN, Cook DG. Short stature, lung function and risk of a heart attack. Int J Epidemiol. 1989; 18:602-606. [PubMed: 2807663]

16. Chae CU, Pfeffer MA, Glynn RJ, Mitchell GF, Taylor JO, Hennekens CH. Increased pulse pressure and risk of heart failure in the elderly. JAMA. 1999; 281:634-639. [PubMed: 10029125]

17. Kouda K, Nakamura H, Fan W, Takeuchi H. Negative relationships between growth in height and levels of cholesterol in puberty: a 3-year follow-up study. Int J Epidemiol. 2003; 32:1105-1110. [PubMed: 14681284]

18. l'Allemand D, Eiholzer U, Schlumpf M, Steinert H, Riesen W. Cardiovascular risk factors improve during 3 years of growth hormone therapy in Prader-Willi syndrome. Eur J Pediatr. 2000; 159:835-842. [PubMed: 11079197]

19. Levy D, Larson MG, Vasan RS, Kannel WB, Ho KK. The progression from hypertension to congestive heart failure. JAMA. 1996; 275:1557-1562. [PubMed: 8622246]

20. Kannel WB, Seidman JM, Fercho W, Castelli WP. Vital capacity and congestive heart failure. The Framingham study. Circulation. 1974; 49:1160-1166. [PubMed: 4275471]

21. Bahrami H, Kronmal R, Bluemke DA, Olson J, Shea S, Liu K, Burke GL, Lima JA. Differences in the incidence of congestive heart failure by ethnicity: the multi-ethnic study of atherosclerosis. Arch Intern Med. 2008; 168:2138-2145. [PubMed: 18955644] 
22. Ridker PM, Danesh J, Youngman L, Collins R, Stampfer MJ, Peto R, Hennekens CH. A prospective study of Helicobacter pylori seropositivity and the risk for future myocardial infarction among socioeconomically similar U.S. men. Ann Intern Med. 2001; 135:184-188. [PubMed: 11487485]

23. Pool PE, Braunwald E. Fundamental mechanisms in congestive heart failure. Am J Cardiol. 1968; 22:7-15. [PubMed: 4232344]

24. Berne, RMWW.; Nelson, MT.; Berne, RM.; Levy, MN. Cardiovascular Physiology Mosby. 2010.

25. Langou RA, Wolfson S, Olson EG, Cohen LS. Effects of orthostatic postural changes on myocardial oxygen demands. Am J Cardiol. 1977; 39:418-421. [PubMed: 842462]

26. Smulyan H, Marchais SJ, Pannier B, Guerin AP, Safar ME, London GM. Influence of body height on pulsatile arterial hemodynamic data. J Am Coll Cardiol. 1998; 31:1103-1109. [PubMed: 9562014] 
Table 1

Baseline characteristics of 22, 042 Male Physicians according to categories of height.

\begin{tabular}{lcccc}
\hline & \multicolumn{5}{c}{ Categories of Height(m) } \\
\hline Characteristics & $\begin{array}{c}\mathbf{1 . 4 0 - 1 . 7 3} \\
(\mathbf{n = 5 6 3 6})\end{array}$ & $\begin{array}{c}\mathbf{1 . 7 4 - 1 . 7 8} \\
(\mathbf{n = 5 9 2 1})\end{array}$ & $\begin{array}{c}\mathbf{1 . 7 9 - 1 . 8 3} \\
(\mathbf{n = 6 5 6})\end{array}$ & $\begin{array}{c}\mathbf{1 . 8 4 - 2 . 0 8} \\
(\mathbf{n = 3 9 1 9})\end{array}$ \\
\hline Height (m) & $1.70(0.04)$ & $1.77(0.01)$ & $1.82(0.01)$ & $1.88(0.03)$ \\
Weight (kg) & $71.29(8.45)$ & $77.29(8.43)$ & $81.79(9.16)$ & $87.91(10.20)$ \\
Age (years) & $54.88(10.35)$ & $54.41(9.55)$ & $53.23(9.21)$ & $52.10(8.57)$ \\
Prevalent Atrial Fibrillation & $0.87 \%$ & $1.62 \%$ & $1.74 \%$ & $2.09 \%$ \\
Prevalent Left Ventricular Hypertrophy & $0.34 \%$ & $0.25 \%$ & $0.11 \%$ & $0.18 \%$ \\
Prevalent Valvular Heart Disease & $0.18 \%$ & $0.17 \%$ & $0.15 \%$ & $0.18 \%$ \\
Prevalent Diabetes Mellitus & $3.67 \%$ & $3.01 \%$ & $2.86 \%$ & $2.27 \%$ \\
Prevalent Coronary Artery Bypass Grafting & $0.73 \%$ & $0.54 \%$ & $0.56 \%$ & $0.54 \%$ \\
Vigorous Exercise & $67.60 \%$ & $72.06 \%$ & $74.29 \%$ & $75.81 \%$ \\
Cigarette smoker & & & & \\
Never & $50.31 \%$ & $49.05 \%$ & $49.63 \%$ & $49.23 \%$ \\
Past & $39.15 \%$ & $39.38 \%$ & $39.13 \%$ & $39.94 \%$ \\
Current & $10.54 \%$ & $11.56 \%$ & $11.24 \%$ & $10.83 \%$ \\
Alcohol use & & & & \\
Rarely & $22.51 \%$ & $25.51 \%$ & $25.00 \%$ & $27.23 \%$ \\
Monthly & $47.14 \%$ & $48.91 \%$ & $50.25 \%$ & $50.06 \%$ \\
Weekly & $12.91 \%$ & $11.31 \%$ & $10.34 \%$ & $9.79 \%$ \\
Daily & $17.43 \%$ & $14.27 \%$ & $14.41 \%$ & $12.92 \%$ \\
Hypertension or on hypertensive medication & $25.59 \%$ & $24.80 \%$ & $23.30 \%$ & $20.68 \%$ \\
\hline
\end{tabular}

Abbreviations: Kg-kilograms, m-meters, SD-Standard deviation

* Data are presented as means (SD) or percentage 
